\title{
A DECISION-MAKING FRAMEWORK BASED ON THE PROSPECT THEORY UNDER AN INTUITIONISTIC FUZZY ENVIRONMENT
}

\author{
Jing GU${ }^{1}$, Zijian $\mathrm{WANG}^{2}$, Zeshui XU ${ }^{3}$, Xuezheng $\mathrm{CHEN}^{4^{*}}$ \\ ${ }^{1}$ School of Economics, Sichuan University, 29 Wangjiang Road, Chengdu, Sichuan, China \\ ${ }^{2}$ School of Statistics and Management, Shanghai University of Finance and Economics, \\ 777 Guoding Road, Shanghai, China \\ ${ }^{3}$ Business School, Sichuan University, 29 Wangjiang Road, Chengdu, Sichuan, China \\ ${ }^{4}$ School of Economics, Jiangxi University of Finance and Economics, \\ No. 169 East Shuanggang Road, Nanchang, Jiangxi, China
}

Received 14 August 2017; accepted 20 May 2018

\begin{abstract}
Uncertainty and ambiguity are prevalent in daily decision-making processes. By closely integrating the prospect theory and the intuitionistic fuzzy sets, this paper develops a generalized framework of decision-making. Theoretically, we first use a four-step editing phase and a valuation phase to generate two key functions: the value function and the weighting function. We then demonstrate how to compute the intuitionistic fuzzy prospect values as the reference for decisionmaking. Empirically, experiments are conducted to examine how well the patterns of subjects' decision-making, under an intuitionistic fuzzy environment, comfort to the framework proposed in this paper. The experiments reveal that the ways that subjects choose to deal with information are consistent with what are implied by the fuzzy logic of our framework in various scenarios. The experimental results show that (i) the shapes of the value function and the weighting function in our framework are in line with those of prospect theory, (ii) people are less risk averse when making decisions under an intuitionistic fuzzy environment than under uncertainty, and (iii) with the presence of ambiguity, people systemically underweight the probabilities of the outcomes with gains as well as those of the outcomes with losses. This paper illustrates that our methodology can elicit prospects not only under uncertainty but also under ambiguity, and the decision-making patterns can be fully captured by parameters in the value function and the weighting function. As our approach generalizes to the case of ambiguity, the prospect theory can therefore be viewed as a special case in our framework.
\end{abstract}

Keywords: decision processes, prospect theory, intuitionistic fuzzy set, intuitionistic fuzzy prospect value.

JEL Classification: A12, C15, C80, E22.

${ }^{\star}$ Corresponding author. E-mail: hymacmechen@hotmail.com

This is an Open Access article distributed under the terms of the Creative Commons Attribution License (http://creativecommons. $\mathrm{org} / \mathrm{licenses} / \mathrm{by} / 4.0 /$ ), which permits unrestricted use, distribution, and reproduction in any medium, provided the original author and source are credited. 


\section{Introduction}

Von Neumann and Morgenstern (1953) develop the expected utility theory (Henceforth EUT), a classic model of rational choice, to illustrate how individuals make decisions under uncertainty. Nonetheless, the Allais paradox, a choice problem designed in Allais (1953), shows an inconsistency of actual observed choices with the predictions of EUT. EUT cannot fully explain how individuals make decisions in certain risky situations, especially when there is fuzzy information, nor can it predict what decisions will be made in such circumstances. Furthermore, EUT cannot explain how framing effect affects individual decisions, or why individuals show risk-seeking or risk-averse behaviors in different circumstances.

To address the above issues, Kahneman and Tversky (1979) develop the prospect theory (Henceforth PT) as an alternative method to explain the choices of people under risk. Kahneman and Tversky points out that the choices made by individuals in risky situations show several characteristics that are inconsistent with EUT, such as certainty effect, isolation effect and reflection effect. These three effects are the main results in PT. Certainty effect means that individuals usually underweight uncertain outcomes in comparison with outcomes that are certain, which leads to risk-aversion in choices involving certain gains and risk-seeking in choices involving certain losses. Isolation effect implies that when facing choices with different prospects, individuals usually disregard components that are common to all prospects under consideration. This effect will cause the framing of a prospect to change the choice that the individual decision-maker makes. Reflection effect means that the choices with negative prospects are mirror images of those ones with positive prospects. Based on the value function and the weight function, we can explain why there exists a framing effect in decisionmaking. As a descriptive model, PT is an important advance in the study of decision-making under risk. It has some significant advantages over the normative EUT model, especially in the study of bounded rationality (Newman, 1980; Payne, Laughhunn \& Crum, 1984; Tversky \& Kahneman, 1986; Edwards, 1996). The main conclusions of PT have been verified in a lot of literature, including Leclerc, Schmitt, and Dube (1995), Sebora and Cornwall (1995), Mayer (1995), and Rieger, Wang, and Hens (2015).

Many researchers reexamine or extend PT in different ways. For example, Tversky and Fox (1995) discuss the relationship between probability judgments and decision weights, and distinguish relative sensitivity from ambiguity aversion. González and Wu (1999) analyze two characteristics of weighting function, discriminability and attractiveness, and construct a new form of the weighting function. M. Levy and H. Levy (2002) develop an improved form of the value function using the PT and prospect stochastic dominance.

Accurate information, however, is often unavailable due to missing data or the lack of preciseness when collecting data. Outliers or inaccurate numbers may induce people to make bad decisions. It turns out that information quality accounts for the evaluation of our behavior. When it is hard to guarantee the information accuracy, it is wise to interpret and turn information with uncertainties and ambiguities into available data. For example, we can use fuzzy information to instruct our decision-making process.

Zadeh (1965) introduces fuzzy sets and fuzzy logic and defines a fuzzy set as a set of membership function, which assigns a grade of membership ranging between zero and one 
to each object. His framework has been widely applied in decision-making under a fuzzy environment. Atanassov (1986) extends the fuzzy set to the membership function of the intuitionistic fuzzy set (IFS), which is characterized by a membership function, a non-membership function and a hesitancy function. Atanassov and Gargov (1989) develop the concept of interval-valued intuitionistic fuzzy set (IVIFS) based on IFS. IFS and IVIFS can describe the fuzzy characteristics of events more comprehensively and they are more effective in dealing with vagueness and uncertainty. Liu and Wang (2007) propose new methods for solving multi-criteria decision-making problems under a fuzzy environment. Xu and Xia (2011) define a series of operators of intuitionistic fuzzy values, which aggregate the intuitionistic fuzzy information and simplify the results of information analysis.

There has been a large literature integrating PT and fuzzy theory into their decisionmaking approaches. Using the trapezoidal fuzzy numbers, Wang and Zhang (2009) propose a PT-based multi-attribute fuzzy decision-making method, which enables people to make decisions by ranking the score values of the trapezoidal fuzzy numbers. It is implied that the choice of $\tilde{a}_{2}$ is a promising one. Liu, Jin, Zhang, Su, and Wang (2011) introduce a PTbased decision-making framework to deal with interval probability and uncertain linguistic variables expressed as triangular fuzzy numbers. Wang and Nie (2012) integrate the value function in PT into the score function of IFS to increase the flexibility of decision-making method. Li, Liu, and Zhu (2012) develop a dynamic stochastic decision-making method and an intuitionistic fuzzy stochastic multi-attribute decision-making method based on PT. Krohling and Souza (2012) apply trapezoidal fuzzy number in multi-criteria decision making problems. Li (2013) develops an intuitionistic trapezoidal fuzzy multi-attribute decisionmaking method based on the cumulative PT and the Choquet integral. Li and Chen (2014) extend the TOPSIS (technique for order preference by similarity to an ideal solution) method, based on PT and the trapezoidal intuitionistic fuzzy numbers for group decision-making. Andrade, González, Fernández, and Gutiérrez (2014) revisit an experiment examining PT using the concept of Compensatory Fuzzy Logic. Meng, Tan, and Chen (2015) introduce a method for multi-attribute decision-making with Atanassov's interval-valued intuitionistic fuzzy information based on PT. Recently, Gao and Liu (2016) introduce a new prospect projection method for interval-valued intuitionistic fuzzy numbers to deal with multi-criteria decision-making problems. Li, Yang, and Wei (2017) construct a new score function for intuitionistic fuzzy numbers, and aggregate the decision-making information in different natural states according to PT.

In summary, the main contributions, of the existing works that integrate PT and fuzzy theory into their decision-making approaches, mainly lie in the following three aspects: a) providing alternative explanations to the Allais paradox; b) extending the application of value functions to the analysis of decision-making under a fuzzy environment; c) enriching the frameworks for decision-making under a fuzzy environment. One of the major limitations of the existing frameworks is that each of them only focuses on certain specific fuzzy attributes or operators of decision-making, such as the intuitionistic trapezoidal fuzzy multi-attribute (Krohling \& Souza, 2012; Li, 2013). To the best of our knowledge, there is no literature that provides a generalized decision-making framework based on PT under an intuitionis- 
tic fuzzy environment, and also establishes a quantitative measurement of decision making procedure. Moreover, there is a lack of literature introducing a unified framework to explain the differences between the decision-making without fuzzy information and that with fuzzy information.

To address the above problem, we firstly develop a generalized decision-making framework based on PT under an intuitionistic fuzzy environment, by closely integrating the PT and the intuitionistic fuzzy sets into our framework. We fully consider the incompleteness of information, which inspires the idea of intuitionistic fuzzy approach to edit incomplete information. It is simple but efficient to integrate fuzzy information not only into value function but also into weighting function in our decision-making procedure. If the information is complete, by setting the indeterminacy degree in intuitionistic fuzzy sets to zero, our decision-making framework degenerates to a traditional PT decision-making method. Thus, we extend PT to a more flexible fuzzy environment setting.

Secondly, our decision-making framework applies PT in an intuitionistic fuzzy environment and demonstrates how to compute the intuitionistic fuzzy prospect value (Henceforth IFPV). In our framework, the IFPV is the reference value for decision-making in an intuitionistic fuzzy environment, and the option with the highest IFPV is the most desirable choice for the decision makers. It is convenient to calculate by aggregating the weights of the given fuzzy information and the potential values of different choices plus a fuzzy punish terms. We will directly rank the desirability of available choices by calculating their corresponding IFPVs. Given the validity and the applicability of our framework, we can always describe a decision-making event by a set of values incorporating the fuzzy information. And then we can calculate and rank the IFPVs of available choices, then decide which is the most promising. The analysis of the experimental results shows that our decision-making framework is illuminating and effective under an intuitionistic fuzzy environment. This paper elaborates a four-step editing phase to process the information and a valuation phase with two key functions to facilitate its application under an intuitionistic fuzzy environment. Then we adapt the value function and the weighting function in our framework to an intuitionistic fuzzy environment and show that their shapes are in line with those of PT. Our framework is not only an extension of PT but also an application of the theory of intuitionistic fuzzy sets in a more general environment.

Thirdly, the experiment reveals surprisingly that people are less risk averse when making decisions under an intuitionistic fuzzy environment than under uncertainty. When adding fuzzy information for the event, people tend to be risk seeking on the fuzzy part. However, people tend to underestimate the events with low possibilities, which is in line with the predictions of PT.

The remainder of the paper is organized as follows: Section 2 discusses those theories closely related to our framework and presents a series of basic formulas and properties of these theories. Section 3 extends PT to construct a decision-making framework under an intuitionistic fuzzy environment and describes the qualitative attributes of the framework. Section 4 carries out experiments to test our framework. Section 5 makes some discussions and concludes the paper. 


\section{Preliminaries}

\subsection{Prospect theory}

Tversky and Kahneman (1992) develop a two-phase model for simple prospects with monetary outcomes. The first is the editing phase and the second is the evaluation phase. During the editing phase, there are major sequential operations: coding, combination, segregation, and cancellation. Coding involves the setting of a reference point by the decision maker, from which all gains and/or losses are measured. Combination consists of aggregation of probabilities associated with identical outcomes. Segregation involves separating the risk components of a prospect from its risk-free components. Cancellation involves discarding all the common components of the prospects.

The main features of the evaluation phase of PT lie in the following aspects:

1) People make decisions based on changes in wealth rather than on the total wealth.

2) The value function $v(x)$, a modification of expected utility function, is S-shaped. $x$ denotes gains or losses. $v^{\prime}(x)>0$ for all $x \neq 0, v^{\prime \prime}(x)>0$ for $x<0$, and $v^{\prime \prime}(x)<0$ for $x>0$. Here, we take 0 as a reference point. There are gains if $x>0$, and loses if $x<0$. It is steeper for the part of losses than for the part of gains (see Figure 1(a)).

3) The weighting function $\pi(p)$, that associates the decision weight with the probability $p$, is a subjective weight of an event. Here, probabilities do not objectively affect the result, but the result is subjectively influenced by the function $\pi(p)$ defined by the decision maker, $\pi^{\prime}(p)>0, \pi^{\prime \prime}(p)>0, \pi(0)=0.1$, and $\pi(1)=0.9$ (see Figure $1(\mathrm{~b})$ ).

4) The framing of alternative outcomes may strongly affect subjects' choices (Tversky \& Kahneman, 1986).

When it is a binary decision, the expected value is an individual's subjective value, which is denoted by $V(x, p ; y, q)=v(x) \pi(p)+v(y) \pi(q)$, where $x, y$ stand for gains or losses respectively, $p, q$ are the corresponding probabilities. When it is a multipleoption decision, an individual's subjective value is the aggregation of weighted values: $V\left(x_{1}, \ldots, x_{m}, p_{1}, \ldots, p_{m} ; y_{1}, \ldots, y_{n}, q_{1}, \ldots, q_{n}\right)=\sum_{i=1}^{m} v\left(x_{i}\right) \pi\left(p_{i}\right)+\sum_{j=1}^{n} v\left(y_{j}\right) \pi\left(q_{j}\right), \quad$ where $x_{1}, \ldots, x_{m}$ represent the gains, $y_{1}, \ldots, y_{n}$ stand for the loss, $p_{1}, \ldots, p_{m}$ and $q_{1}, \ldots, q_{n}$ are their corresponding probabilities.

\subsection{The theory of intuitionistic fuzzy sets}

In order to encode the information, we first introduce the intuitionistic fuzzy set (IFS). Let $X$ be a set of consequences. Atanassov (1986) defines the IFS as: $A=\left\{\left\langle x, \mu_{A}(x), \sigma_{A}(x)\right\rangle \mid x \in X\right\}$, which assigns to each consequence $x$ a membership degree $\mu_{A}(x)$ and a non-membership degree $\sigma_{A}(x)$, with the condition: $0 \leq \mu_{A}(x)+\sigma_{A}(x) \leq 1, x \in X$. In addition, $\pi_{A}(x)=1-\mu_{A}(x)-\sigma_{A}(x)$ is called the indeterminacy degree of $x$ to $A$. For for the sake of simplicity, we use the intuitionistic fuzzy value (IFV) (Xu \& Cai, 2012) $\alpha$ to denote each component of the IFS: $\alpha=\left(\mu_{\alpha}(x), \sigma_{\alpha}(x)\right)$. Here, $\mu_{\alpha}(x) \in[0,1], \sigma_{\alpha}(x) \in[0,1]$ and $\mu_{\alpha}(x)+\sigma_{\alpha}(x) \leq 1$. Additionally, $\pi_{\alpha}(x)=1-\mu_{\alpha}(x)-\sigma_{\alpha}(x)$. There is a specific meaning for each IFV. For example, if $\alpha=(0.5,0.3)$, then it can be interpreted as " 10 -members voting in favor of resolutions 5, 3 objections, and 2 abstentions." 
a)

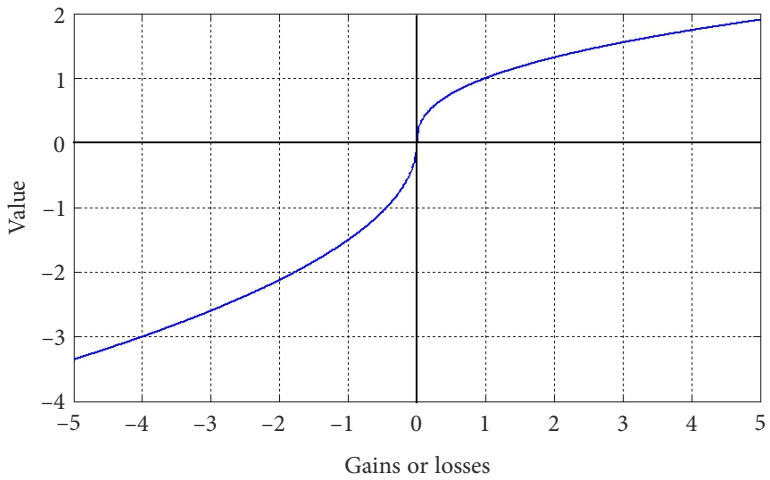

b)

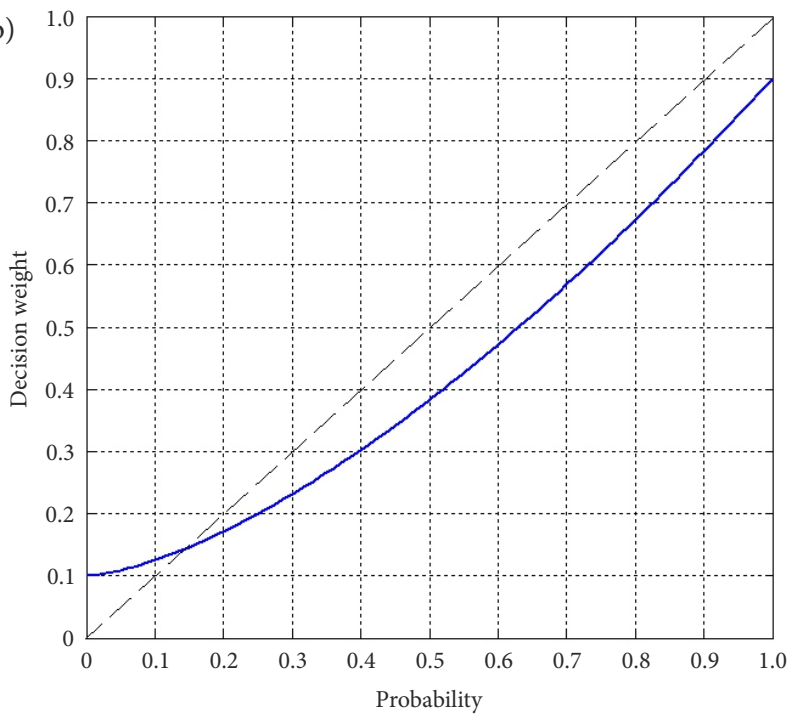

Figure 1. The value function and the weighting function: a - the value function; $\mathrm{b}$ - the weighting function

Here we introduce some basic operational laws of IFVs (Xu \& Cai, 2012), which will be used when coding the information. Suppose $\alpha=\left(\mu_{\alpha}, \sigma_{\alpha}\right), \alpha_{1}=\left(\mu_{\alpha_{1}}, \sigma_{\alpha_{1}}\right)$ and $\alpha_{2}=\left(\mu_{\alpha_{2}}, \sigma_{\alpha_{2}}\right)$ are three IFVs, then:

(1) $\bar{\alpha}=\left(\mu_{\alpha}, \sigma_{\alpha}\right)$;

(2) $\alpha_{1} \oplus \alpha_{2}=\left(\mu_{\alpha_{1}}+\mu_{\alpha_{2}}-\mu_{\alpha_{1}} \mu_{\alpha_{2}}, \sigma_{\alpha_{1}} \sigma_{\alpha_{2}}\right)$;

(3) $\alpha_{1} \otimes \alpha_{2}=\left(\mu_{\alpha_{1}} \mu_{\alpha_{2}}, \sigma_{\alpha_{1}}+\sigma_{\alpha_{2}}-\sigma_{\alpha_{1}} \sigma_{\alpha_{2}}\right)$;

(4) $\lambda \alpha=\left(1-\left(1-\mu_{\alpha}\right)^{\lambda}, \sigma_{\alpha}^{\lambda}\right), \lambda>0$;

(5) $\alpha^{\lambda}=\left(\mu_{\alpha}^{\lambda}, 1-\left(1-\sigma_{\alpha}\right)^{\lambda}\right), \lambda>0$. 
Let $S_{p}\left(\mu_{\alpha_{1}}, \mu_{\alpha_{2}}\right)=\mu_{\alpha_{1}}+\mu_{\alpha_{2}}-\mu_{\alpha_{1}} \mu_{\alpha_{2}}$ and $T_{p}\left(\sigma_{\alpha_{1}}, \sigma_{\alpha_{2}}\right)=\sigma_{\alpha_{1}} \sigma_{\alpha_{2}}$, then the operational law (2) can be rewritten as:

$$
\alpha_{1} \oplus \alpha_{2}=\left(S_{p}\left(\mu_{\alpha_{1}}, \mu_{\alpha_{2}}\right), T_{p}\left(\sigma_{\alpha_{1}}, \sigma_{\alpha_{2}}\right)\right) .
$$

Let $S_{p}\left(\sigma_{\alpha_{1}}, \sigma_{\alpha_{2}}\right)=\sigma_{\alpha_{1}}+\sigma_{\alpha_{2}}-\sigma_{\alpha_{1}} \sigma_{\alpha_{2}}$ and $T_{p}\left(\mu_{\alpha_{1}}, \mu_{\alpha_{2}}\right)=\mu_{\alpha_{1}} \mu_{\alpha_{2}}$, then the operational law (3) can be rewritten as:

$$
\alpha_{1} \otimes \alpha_{2}=\left(T_{p}\left(\mu_{\alpha_{1}}, \mu_{\alpha_{2}}\right), S_{p}\left(\sigma_{\alpha_{1}}, \sigma_{\alpha_{2}}\right)\right),
$$

where $S_{p}\left(x_{1}, x_{2}\right)=x_{1}+x_{2}-x_{1} x_{2}$ is a well-known t-conorm, and $T_{p}\left(y_{1}, y_{2}\right)=y_{1} y_{2}$ is a wellknown t-norm. Both satisfy the properties: boundary, monotonicity, commutativity and associativity. These operational laws are vital in our framework. The economic implications of these operational laws will be explained later.

In many real-world decision-making problems, the values of the membership function and the non-membership function in an IFS are difficult to be expressed as specific numbers. Instead, we can only roughly know the ranges of their values. To address this problem, Atanassov and Gargov (1989) extend the concept of IFS to interval-valued intuitionistic fuzzy set (IVIFS), and define some basic operational laws of IVIFSs. Xu (2007) defines the concept of interval-valued intuitionistic fuzzy value (IVIFV) and specify some basic operational laws of IVIFVs. He puts forward the interval-valued intuitionistic fuzzy weighted average operator and the interval-valued intuitionistic fuzzy weighted geometric operator, and defines the score function and the accuracy function of IVIFVs. All these aggregation techniques for interval-valued intuitionistic fuzzy information are the generalizations of the above intuitionistic fuzzy aggregation techniques.

Let $X$ be a fixed set. Then $\tilde{A}=\left\{\left\langle x, \tilde{\mu}_{\tilde{A}}(x), \tilde{v}_{\tilde{A}}(x)\right\rangle \mid x \in X\right\}$ is called an interval-valued intuitionistic fuzzy set (IVIFS), where $\tilde{\mu}_{\tilde{A}}(x) \subset[0,1]$ and $\tilde{v}_{\tilde{A}}(x) \subset[0,1], x \in X$, with the condition: $\sup \tilde{\mu}_{\tilde{A}}(x)+\sup \tilde{v}_{\tilde{A}}(x) \leq 1, x \in X$. We must note that $\tilde{\mu}_{\tilde{A}}(x)$ and $\tilde{v}_{\tilde{A}}(x)$ mean the interval-valued membership degree and the interval-valued non-membership degree of $x$ in $\tilde{A}$ repectively. Clearly, if $\inf \tilde{\mu}_{\tilde{A}}(x)=\sup \tilde{\mu}_{\tilde{A}}(x)$ and $\inf \tilde{v}_{\tilde{A}}(x)=\sup \tilde{v}_{\tilde{A}}(x)$, then the IVIFS $\tilde{A}$ can be reduced to a traditional IFS.

For convenience, an IVIFV is generally simplified as $(\mathrm{Xu}, 2007): \tilde{\alpha}=([a, b],[c, d])$, where $[a, b] \subset[0,1],[c, d] \subset[0,1], b+d \leq 1$. Obviously, $\tilde{\alpha}^{+}=([1,1],[0,0])$ is the largest IVIFV, and $\tilde{\alpha}^{-}=([0,0],[1,1])$ is the smallest IVIFV. In particular, if $\tilde{\alpha}_{1}=\left(\left[a_{1}, b_{1}\right],\left[c_{1}, d_{1}\right]\right)$ and $\tilde{\alpha}_{2}=\left(\left[a_{2}, b_{2}\right],\left[c_{2}, d_{2}\right]\right)$ are IVIFVs, then $\tilde{\alpha}_{1}=\tilde{\alpha}_{2}$ if and only if $a_{1}=a_{2}, b_{1}=b_{2}, c_{1}=c_{2}$ and $d_{1}=d_{2}$.

Based on the definition of IVIFV, it is easy to derive the following basic operational rules of IVIFVs:
(1) $\overline{\tilde{\alpha}}=([c, d],[a, b])$;
(2) $\tilde{\alpha}_{1} \oplus \tilde{\alpha}_{2}=\left(\left[a_{1}+a_{2}-a_{1} a_{2}, b_{1}+b_{2}-b_{1} b_{2}\right],\left[c_{1} c_{2}, d_{1} d_{2}\right]\right)$;
(3) $\tilde{\alpha}_{1} \otimes \tilde{\alpha}_{2}=\left(\left[a_{1} a_{2}, b_{1} b_{2}\right],\left[c_{1}+c_{2}-c_{1} c_{2}, d_{1}+d_{2}-d_{1} d_{2}\right]\right)$;
(4) $\lambda \tilde{\alpha}=\left(\left[1-(1-a)^{\lambda}, 1-(1-b)^{\lambda}\right],\left[c^{\lambda}, d^{\lambda}\right]\right), \lambda>0$;
(5) $\tilde{\alpha}^{\lambda}=\left(\left[a^{\lambda}, b^{\lambda}\right],\left[1-(1-c)^{\lambda}, 1-(1-d)^{\lambda}\right]\right), \lambda>0$. 


\section{Constructing the intuitionistic fuzzy decision-making framework based on Prospect Theory}

Many existing works focus on the events without ambiguous information. For instance, a question related to $\mathrm{PT}$ is:

Which of the following do you prefer?

A) $50 \%$ chance to win $\$ 1000.50 \%$ chance to win nothing; B) $\$ 450$ for sure.

In reality, however, we may not be able to know the chance of winning and/or losing precisely, instead, we merely have fuzzy information about the probabilities. Sometimes, the conceived probability of an event may be just an arbitrary method to describe the uncertainty. In addition, we may not be able to pinpoint the exact value of actual gains or losses. For example, after evaluating an asset, we find that the return could be about $\$ 1,000$. Here, how to quantify "about" is sometimes very important in quantitative analysis. We may have a piece of information indicating that the return on your asset is approximately $\$ 995$ to $\$ 1,005$. This is one way to express fuzzy information. In this paper, we will present a framework to express fuzzy information in a proper way. In the field of intuitionistic fuzzy sets, Xu and Xia (2011) use intuitionistic fuzzy information to describe the uncertainty of events.

In this paper, we aim to develop a decision-making framework that applies PT under an intuitionistic fuzzy environment. We use fuzzy method to encode information sets, and denote the intuitionistic fuzzy sets as independent variables in constructing our decisionmaking framework. We modify the value function and the weighting function in PT to fit for the decision-making environment with fuzzy information.

The selection analysis in our framework involves two phases: the editing phase and the evaluation phase. In the editing phase, we use the definitions and operators of the intuitionistic fuzzy sets in Xu and Cai (2012) to interpret contingencies and outcomes. Then, in the valuation phase, the decision makers evaluate the prospect values of different choices based on the aggregation functions under an intuitionistic fuzzy environment.

\subsection{Editing phase}

In the editing phase, we organize and re-formulate the options, to simplify subsequent evaluation and decision-making. The editing phase involves the following four steps: coding, combination, scoring and correction.

\subsubsection{Coding}

Based on this intuitionistic fuzzy norm, we interpret fuzzy information more specifically in an investment event. First, we take IFVs as an example. Let $Z$ be a finite set of natural states, the subsets of $Z$ are called events. In this paper, $X$ is a set of consequences or outcomes measured by monetary payoffs. We assume that 0 is included in $X$ as a neutral outcome. While all other $x \in X$ are either gains or losses, expressed as positive or negative numbers respectively. Then the prospect $f$ is a binary function. For each state $z_{i} \in Z$, where $-m \leq i \leq n$, and an IFV $\alpha_{i}$, there is a consequence $f\left(z_{i}, \alpha_{i}\right)=x_{i}$. Here we arrange $x_{i} \in X$ in a monotonically increasing order, i.e., $x_{i}>x_{j}$ iff $i>j$. 
Here, how do we get $\alpha_{i}$, the fuzzy degree of the event $z_{i}$ ? We introduce a scoring system to represent $\alpha_{i}$, which could be based on some the evaluation of an expert. In making investment decisions, we often need to consider the information from various sources. The acquisition process of $\alpha_{i}$ is described in Section 3.1.4. For the sake of simplicity, a prospect $f$ can be expressed as a sequence of a pair $\left(x_{i}, \alpha_{i}\right)$, which means the consequence $x_{i}$ has characteristic of $\alpha_{i}$, where $\alpha_{i}=\left(\mu_{\alpha}\left(x_{i}\right), \sigma_{\alpha}\left(x_{i}\right)\right)=\left(\mu_{i}, \sigma_{i}\right)$. Thus, the prospect $f_{i}=\left(x_{i}, \alpha_{i}\right)$ describes the situation where the outcome $x_{i}$ will occur at about probability of $\mu_{i}$, and will not occur at about probability of $\alpha_{i}$. In addition, the outcome $x_{i}$ has a hesitance value of $\pi_{i}$. It shows the fuzzy degree of the outcome, which means that it may occur or may not occur without any specific information. Here, $0 \leq \mu_{i} \leq 1,0 \leq \sigma_{i} \leq 1$, and $0 \leq \mu_{i}+\sigma_{i} \leq 1, i=-m, \ldots, n$.

\subsubsection{Combination}

Now we define the combinatorial principle in the case of fuzzy information. There is a series of fuzzy events or a series of fuzzy event attributions $z_{i}$. If they have an identical consequence, we can combine them to simplify the decision-making. At the same time, we have the fuzzy numbers for all the events or attributions $\alpha_{i}$, where $-m \leq i \leq n$. Now we are keen to find out how to describe the aggregate consequence or attribution by IFVs. In the process of decisionmaking, we only need to focus on the final consequence, while other events can be negligible. It is therefore necessary to incorporate relevant information. After coding all the events, some information can be aggregated to simplify the decision-making process.

In combination, the addition principle is the basic principle. For example, if we have $a$ ways of doing something and $b$ ways of doing another thing. If we cannot choose more than one way at the same time, then we need to choose an action from the set consisting of $a+b$ ways. According to the set theory, the size of the union sets is the sum of all finite sets, which are pairwise disjoint sets. For the IFS, we can apply this rule as well. If all the events are independent, the sum of the events $z_{i}(i=-m, \ldots, n)$ is represented by $\sum_{i=-m}^{n} \alpha_{i}=\alpha_{-m} \oplus \alpha_{-m+1} \oplus \cdots \oplus \alpha_{n}$. An aggregate event or attribution is still represented by an $i=-m$
IFV. It means that each of these fuzzy events will take place with the information of $\sum_{i=-m}^{n} \alpha_{i}$.
This is the algorithm for parallel events or attributions.

However, if the events are correlated and sequential, then we will use the multiplication principle to calculate the aggregate contribution. For example, if there are $a$ ways of doing something and $b$ ways of doing another thing sequentially, then there are $a \cdot b$ ways of doing both things. Therefore, if the events $z_{i}(i=-m, \ldots, n)$ occur in succession, then we define $\prod_{i=-m}^{n} \alpha_{i}=\alpha_{-m} \otimes \alpha_{-m+1} \otimes \cdots \otimes \alpha_{n}$ the fuzzy characteristic as an aggregate event. This is the algorithm for series of sequential events or attributions.

Based on the above addition principle, multiplication principle and scoring system, we can check any information of any steps at any point of time. The intuition of the above two principles can be illustrated in the following Figure 2 .

In summary, addition principle in fuzzy theory implies that if the fuzzy events are parallel, an aggregate event is represented by the sum of individual IFVs. Multiplication principle in fuzzy theory means that if the fuzzy events is sequential, an aggregated event is repre- 
sented by the product of individual IFVs. For example, there is an engineering project that can be accomplished by different ways. We can use the addition principle of fuzzy theory to figure out the total success rate of this project. As long as one method is successful, this project will be accomplished. Hence, the aggregated success rate is expressed as the sum of all fuzzy numbers that define the chance of success for each mode. Multiplication principle can be adopted to illustrate many events. For example, there is an investment project with many stages, and the success probability for each stage can be expressed as a fuzzy number. In this case, the probability of an success rate of an investment project is represented by the product of all fuzzy numbers.

a)

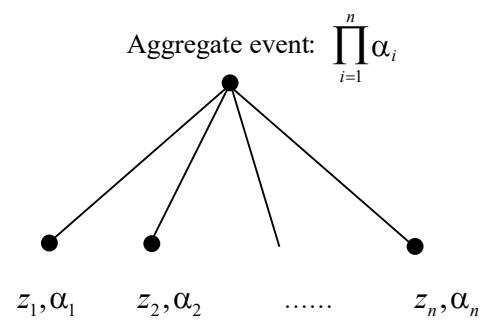

b)

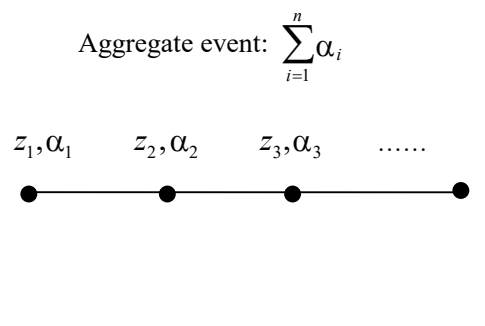

Figure 2. The addition and multiplication principle under fuzzy information: $\mathrm{a}$ - addition principle in fuzzy theory; $\mathrm{b}$ - multiplication principle in fuzzy theory

\subsubsection{Scoring}

Scoring is an effective way of decision-making. First, we gather as much information as possible. For example, when considering whether to invest in the stock market, we would like to refer to some television programs for stock investment, which often recommend some securities or stocks. According to their opinion, we assign a membership score and a nonmembership score between 0 and 1 for each of the securities or stocks, indicating the pros and cons. we can then evaluate the information obtained from the television programs and assign subjective scores. These scores $\alpha_{i}$, where $i=1,2,3 \ldots$, are the intuitionistic fuzzy information. This scoring method is also suitable for multi-attribute decision-making. We first identify all the attributes of a plan/project to be evaluated and then provide a score for each of the attributes to get $\alpha_{i}$. For different situations, the scoring system works in different ways, but we can always obtain the fuzzy information that we need for editing.

\subsubsection{Correction}

Following the scoring step, we may find that some scores are not easy to deal with. Therefore, we make some corrections to the scores, so that the accuracy can be guaranteed within the bounds of an acceptable range. For example, if the original $\alpha=(0.999,0.001)$, we can correct it as $\alpha=(1,0)$ to simplify the information. Moreover, if two events are very closely related, we treat them as an event and assign a proper fuzzy number to this event.

In edition phase, we introduce four steps to edit the regular events or attributions, which cannot be calculated in the form of text description. We use numerical consequences and IFVs or IVIFVs $\left(x_{i}, \alpha_{i}\right)$ to represent the information. Before we evaluate events or attribu- 
tions for decision-making, we can analyze the information in our framework. This method can also be applied to multi-attribute decision-making.

For example, there is a prospect $f$ with two goals. There are three independent ways to achieve the first goal, and two tasks should be accomplished sequentially in order to achieve the second goal. We denote two goals as $z_{1}$ and $z_{2}$, with the consequences $x_{1}$ and $x_{2}$ respectively. We denote the three ways to achieve first goal by $z_{11}, z_{12}, z_{13}$, with the IFVs $\alpha_{11}, \alpha_{12}, \alpha_{13}$ respectively. Two events of the second goal are denoted by $z_{21}$ and $z_{22}$, with the IFVs $\alpha_{21}$ and $\alpha_{22}$ respectively According to the addition principle, $z_{1}$ has fuzzy information $\alpha_{11} \oplus \alpha_{12} \oplus \alpha_{13}=\alpha_{1}$. Then the second goal $z_{2}$ has fuzzy information $\alpha_{21} \otimes \alpha_{22}=\alpha_{2}$. Therefore, the prospect is described as $f=\left(x_{1}, \alpha_{1} ; x_{2}, \alpha_{2}\right)$.

When people make decisions, it is essential to reveal the subjective value of ambiguity. Then we can figure out the actual prospect value of an option in a fuzzy environment. This subjective prospect value is an important basis of decision-making. In many cases, the decision makers are not completely rational, or the information is incomplete or not precise enough. To address the above problem, we develop a decision-making framework based on PT under an intuitionistic fuzzy environment, which extends PT with the information expressed by IFVs or IVIFVs.

\subsection{Valuation phase}

In the valuation phase, we incorporate IFVs or IVIFVs into our value function. Here, we assume that the value function $V(f)$ is monotonically increasing and $V(0)=0$. For a prospect $f=\left(x_{i}, \alpha_{i}\right),-m \leq i \leq n$, we discuss the positive and negative parts separately, denoted by $f^{+}$and $f^{-}$respectively. $f^{+}$represents all the positive outcomes in the prospect $f$, while $f^{-}$represents all the negative outcomes in the prospect $f$. If a prospect consists of outcomes with both gains and losses, we can classify these outcomes into two different prospects, a positive prospect consisting of all outcomes with gains, and a negative prospect consisting of all outcomes with losses. The advantage of doing such a classification is that we can clearly compare the aggregate result of all the outcomes with gains with that of all the outcomes with losses. The IFPV subjective value is defined as follows:

$$
\begin{aligned}
& V(f)=V\left(f^{+}\right)+V\left(f^{-}\right) ; \\
& V\left(f^{+}\right)=\sum_{i=0}^{n}\left(g^{+}\left(s_{i}\right) v\left(x_{i}\right)+\beta^{+} \pi_{i} v\left(x_{i}\right)\right) ; \\
& V\left(f^{-}\right)=\sum_{i=-m}^{0}\left(g^{-}\left(s_{i}\right) v\left(x_{i}\right)+\beta^{-} \pi_{i} v\left(x_{i}\right)\right),
\end{aligned}
$$

where $s_{i}=\mu_{i}-\sigma_{i}$ is the score function of an IFV $\alpha_{i}$ (Chen \& Tan, 1994). $\beta^{+}$and $\beta^{-}$are the parameters of the fuzzy terms in the positive prospect and the negative prospect respectively. Since there is no effective way to test $\beta^{+}$and $\beta^{-}$, we assume that $\beta^{+}$or $\beta^{-}$are random numbers from a normal distribution. $\pi_{i}=1-\mu_{i}-\sigma_{i}$ is the indeterminacy function, and $g(\cdot)$ is the weighting function with the independent variable $s_{i}$, which satisfies the condition $-1 \leq s_{i} \leq 1$. 
$v(x)$ is the value function, which is usually a two-part exponential function expressed as:

$$
v(x)=\left\{\begin{array}{ll}
A x^{p} & \text { if } x \geq 0 \\
-\lambda(-x)^{q} & \text { if } x<0
\end{array},\right.
$$

where $p$ and $q$ in the value function (6) are the risk attitude parameters, $A$ and $\lambda$ are the gain coefficient and the loss aversion coefficient.

The interpretation of the equations (4) and (5) is that the subjective value of the outcome is multiplied by its weighting function. We adopt the score function instead of the probability as an independent variable of the weighting function. Here, we assume that people make decisions based on the net certainty level represented by $s_{i}=\mu_{i}-\sigma_{i}$, rather than the separated probabilities in the existing literature. Both weighting functions, $g^{+}(\cdot)$ and $g^{-}(\cdot)$, are non-linear but strictly increasing in the intervals $[-1,1]$, and satisfy the following two constraints: $g^{+}(-1)=g^{-}(-1)=0$ and $g^{+}(1)=g^{-}(1)=1$. Moreover, we impose a penalty on each total subjective value, measuring the impact of the indeterminacy of the outcomes. Here, $\beta^{+}$ and $\beta^{-}$are constant numbers. Then for every outcome, the subjective value of the event $z_{i}$ is measured by either $g^{+}\left(s_{i}\right) v\left(x_{i}\right)+\beta^{+} \pi_{i} v\left(x_{i}\right)$ or $g^{-}\left(s_{i}\right) v\left(x_{i}\right)+\beta^{-} \pi_{i} v\left(x_{i}\right)$, and the sum of the subjective values of each event represents the overall value of a prospect.

To illustrate the model, let's consider the following game with intuitionistic fuzzy information. You pick up a ball from a large dark urn, which is filled with about 30 white balls and about 50 black balls, and around 20 balls, and we do not know whether they are white or black. The total amount of balls is 100. If you pick up a white ball, there is no reward. However, if you get a black ball, you will be rewarded with 100 USDs. Based on the above information, we can calculate the IFPV of white ball and that of black ball respectively. For white balls, the outcome is 0 USDs with the IFV $\alpha_{\text {white }}=(0.3,0.5)$. For black balls, the outcome is 100 USDs with the IFV $\alpha_{\text {black }}=(0.5,0.3)$. Therefore, the prospect of picking up a ball can be expressed as $f^{+}=(0,(0.3,0.5) ; 100,(0.5,0.3))$. Based on the equation (4), we have:

$$
V\left(f^{+}\right)=g^{+}(-0.2) \cdot v(0)+\beta^{+} \cdot 0.2 \cdot v(0)+g^{+}(0.2) \cdot v(100)+\beta^{+} \cdot 0.2 \cdot v(100) .
$$

This is only an example of positive prospect situation, if the game is to lose 100 USDs who pick up the black ball, then it is an example of negative prospect, expressed as $f^{-}=(0,(0.3,0.5) ;-100,(0.5,0.3))$. Now suppose that we are investing a stock, expected profit is $x$, however, the intuitionistic fuzzy information of this profit is $\alpha_{1}=(0.5,0.3)$, if you don't invest, expected profit is of course 0 . And the prospect of this event is $f_{i}^{+}=(0,(0.3,0.5) ; x,(0.5,0.3))$, and the IFPV of this prospect is calculated in the same way.

So far, we have constructed a basic framework for decision-making under fuzzy information. We can compute the IFPV of each choice and decide the optimal choice based on the ranking of their IFPVs. Figure 3 shows the decision-making process of our framework.

We only consider the fuzzy aspects of the above framework. Nonetheless, both the value function and the weighting function are important in PT framework, and themselves can also be used for the events with fuzzy information. Here, we introduce a modified value function and a modified weighting function under an intuitionistic fuzzy environment. 


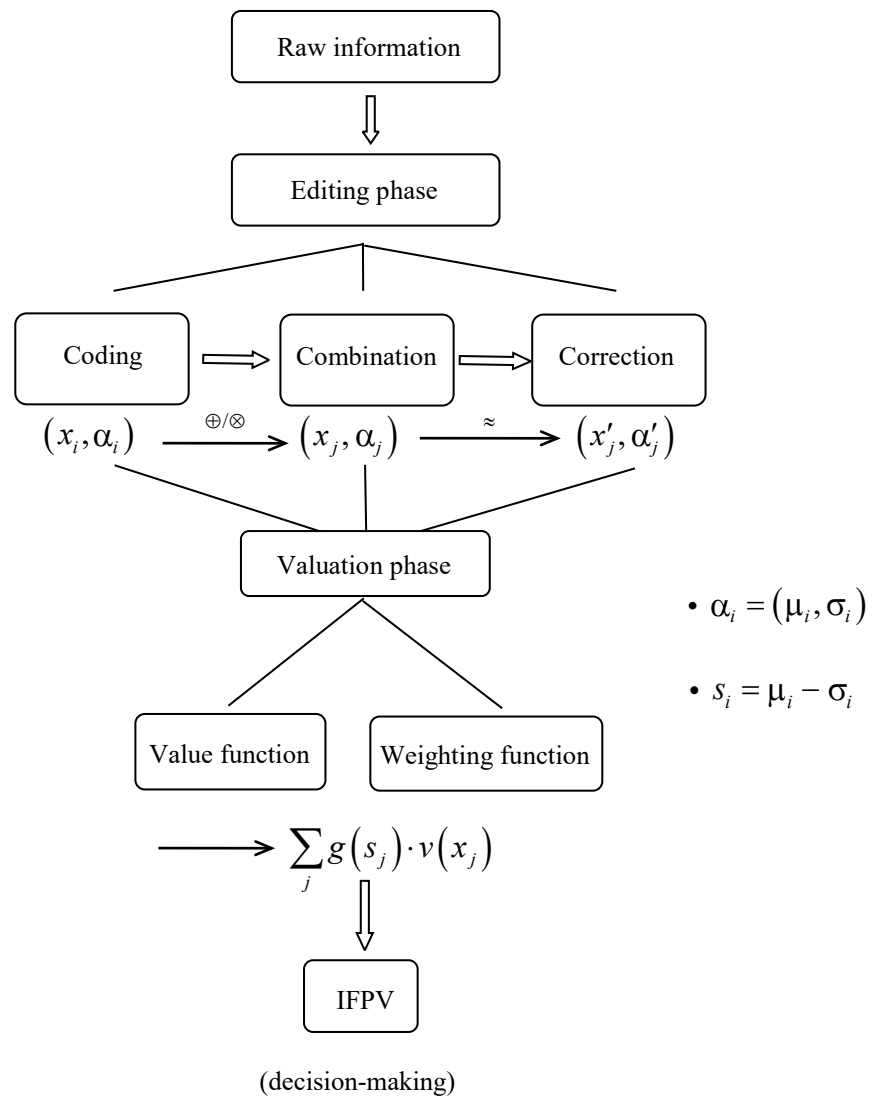

Figure 3. The decision-making framework based on PT under an intuitionistic fuzzy environment

\subsubsection{The value function}

In the following part, we will apply the value function under an intuitionistic fuzzy environment. The value function is determined by the monetary payoff $x_{i}$, however, $x_{i}$ may be an interval-valued number. We assume that the monetary payoff $x_{i}$ is determined by an IVIFV $\tilde{\alpha}_{i}=\left(\left[a_{i}, b_{i}\right],\left[c_{i}, d_{i}\right]\right)$, which means that the actual monetary payoff is close, but not exactly equal to $x_{i}$. We still use the scoring system to decide $\tilde{\alpha}_{i}$. Therefore, the membership value of $x_{i}$ is $\left[a_{i}, b_{i}\right]$, and the non-membership value is $\left[c_{i}, d_{i}\right]$. Then the accuracy function of an IVIFV $\tilde{\alpha}_{i}$ is defined as:

$$
h\left(\tilde{\alpha}_{i}\right)=\frac{1}{2}\left(a_{i}+b_{i}+c_{i}+d_{i}\right) .
$$

The intuition of the accuracy function is with probability $h\left(\tilde{\alpha}_{i}\right)$, the monetary payoff is $x_{i}$. In other word, since $0 \leq h\left(\tilde{\alpha}_{i}\right) \leq 1$, the real monetary payoff is one of the values in the interval defined by:

$$
\left[x_{i}-x_{i} \cdot\left(1-h\left(\tilde{\alpha}_{i}\right)\right), x_{i}+x_{i} \cdot\left(1-h\left(\tilde{\alpha}_{i}\right)\right)\right]=\left[h\left(\tilde{\alpha}_{i}\right) \cdot x_{i},\left(2-h\left(\tilde{\alpha}_{i}\right)\right) \cdot x_{i}\right] .
$$


Here, we assume that the true values are evenly distributed in the interval. This means that the real monetary payoff can be any value in the interval $\left[h\left(\tilde{\alpha}_{i}\right) \cdot x_{i},\left(2-h\left(\tilde{\alpha}_{i}\right)\right) \cdot x_{i}\right]$ with the same possibility. We denote this random number as $x_{i}^{\prime}$. The value function can be expressed as:

$$
v\left(x_{i}, \tilde{\alpha}_{i}\right)=\left\{\begin{array}{llc}
A\left(x_{i}^{\prime}\right)^{p} & \text { if } & x_{i}^{\prime} \geq 0 \\
-\lambda\left(-x_{i}^{\prime}\right)^{q} & \text { if } & x_{i}^{\prime}<0
\end{array}\right.
$$

where $x_{i}^{\prime}$ is a real value or a modification under the interval-valued intuitionistic fuzzy environment, which can be obtained by the method mentioned above.

According to PT and other related studies, the curve of value function has the following three basic features: (1) defined by deviations from the reference point; (2) concave for gains and convex for losses; (3) steeper for losses than for gains.

The value function in our framework is close to that in PT, but the actual scaling is considerably more complex than the original value function. It can increase or offset the curvature. How is the value function shaped in this paper? To answer this question, we will design and carry out experiments in the next section.

\subsubsection{The weighting function}

Decision weights are not equivalent to probabilities in our daily life, so do not obey the probability axioms. Here, we must note that the weighting function is used to reveal the subjective weight of an event, whereas the IVIFV in the value function is used to reveal the vagueness of a nominal value. Although IVIFVs and IFVs are both fuzzy information, their implications are rather different. We adopt the score function of IFVs as the impact factor of decision weight. Here, we assign weights based on the shortfall between the membership and the non-membership. This is because the wealth or welfare change is what we really concern, rather than the final states. Inspired by Gonzalez and Wu (1999), we define the weighting function under an intuitionistic fuzzy environment as:

$$
g^{+}\left(s_{i}\right)=\frac{\eta\left(s_{i}+1\right)^{\gamma}}{\eta\left(s_{i}+1\right)^{\gamma}+\left(1-s_{i}\right)^{\gamma}}, g^{-}\left(s_{i}\right)=\frac{\eta\left(s_{i}+1\right)^{\delta}}{\eta\left(s_{i}+1\right)^{\delta}+\left(1-s_{i}\right)^{\delta}},
$$

where $\eta, \gamma, \delta$ are the constants.

The domain of the original weighting function is $[0,1]$. Nonetheless, in our framework, the domain of the weighting function is $[-1,1]$. Figure 4 shows the shape of the weighting function $g^{+}\left(s_{i}\right)$ with approximate fuzzy information. Here, we let $\eta=0.77$ and $\gamma=0.44$, which are given by Gonzalez and Wu (1999). The weighting function is inverse-S-shaped, which captures the fact that people generally overestimate the low-scoring events and underestimate high-scoring ones. The numerical values here are based on the parameters estimated in PT. When the score of an event is close to -1 or 1 , there is virtually no difference between a normal case and a case with intuitionistic fuzzy information. As shown in the following figure, event scores between -1 and 1 may have a more complex weight distribution. This feature will be examined in our experiments. 


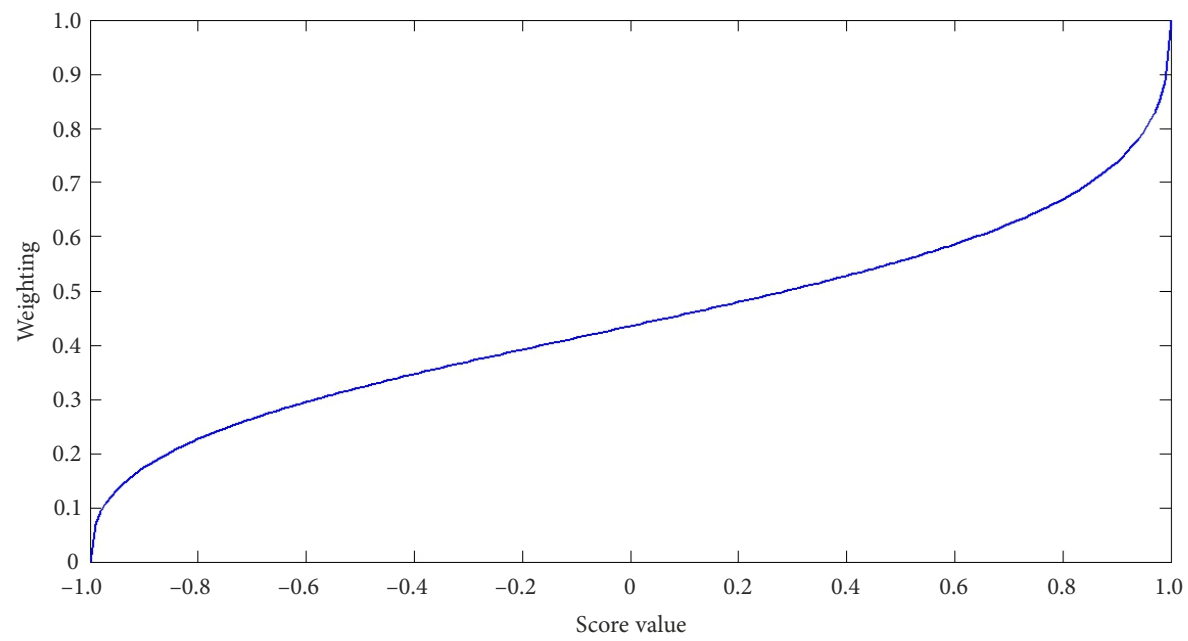

Figure 4. The weighting function with fuzzy information

\subsection{Main properties of our framework}

The followings are the main properties of our decision-making framework:

1) Diminishing sensitivity and reflection effect. We define the reference point as 0 , then $v$ is concave above the reference point (i.e., $\left.v^{\prime \prime}(x) \leq 0, x \geq 0\right)$ and convex below the reference point (i.e., $\left.v^{\prime \prime}(x) \geq 0, x \leq 0\right)$. It is one of the basic properties of PT. This property shows that people become less and less sensitive with the increase in both gains and losses. For example, people are less sensitive to an increase from 100 to 110 USDs than to an increase from 10 to 20 USDs. They are also less sensitive to a decrease from 110 to 100 USDs than to a decrease from 20 to 10 USDs.

2) Loss aversion. $v$ is steeper for losses than for gains, i.e. $v^{\prime}(x)<v^{\prime}(-x)$, for $x \geq 0$. This property indicates that most people are loss aversion, i.e., people tend to prefer avoiding losses to acquiring the same amount of gains.

3) Risk seeking. The weighting functions $g^{+}$and $g^{-}$are both inverse-S functions. When the score value $s$ is close to -1 , the slope of the weighting function is very steep. Intuitively, when faced with small probability events, such as lotteries, people tend to be more risk-seeking.

4) Certainty effect. This means when the score value $s$ is closer to 1 , the weighting function rises faster. The intuition is that when the certainty level of an event becomes sufficiently high, a decision maker's subjective evaluation of the weight will increase in proportion to the increase in its level of certainty.

5) Additivity. If two separate events result in the same outcome $x^{*}$ with different IFVs $\alpha_{1}, \alpha_{2}$. We can use the IFV $\alpha_{1} \oplus \alpha_{2}$ to match this outcome as a new prospect $f^{*}=\left(x^{*}, \alpha_{1} \oplus \alpha_{2}\right)$. It is more convenient to combine two similar outcomes into one when we carry out framing analysis.

6) Multiplicativity. If two events occur sequentially with the IFVs $\alpha_{1}, \alpha_{2}$, and may give rise to an outcome $x^{*}$. We can integrate them by using the multiplication principle. 
IFVs are the expressions of information in our paper. It is worth noting that IFVs are not the same to the probability and improbability of an event. $\mu_{\alpha}$ and $\sigma_{\alpha}$ in $\alpha$ just present the membership degree and the non-membership degree of an event. Therefore, they do not satisfy the probability axioms.

In our frame work, we express the score functions as independent variables of the weighting function. The image of the weighting function shows that our framework is consistent with the certainty effect of PT and the other attributes of decision-making.

\section{Experiments}

In this section, we design experiments to verify our intuitionistic fuzzy PT framework. The first one is to fit the value function and the weighting function. The parameters in both functions are obtained from the experimental results. The second experiment is to verify the addition principle and the multiplication principle, to ensure the accuracy of IFPT framework.

\subsection{Participants}

We invite 12 students randomly, 6 graduates and 6 undergraduates, from the School of Economics at Sichuan University. Participants are asked to fill a questionnaire. We want to find the certain equivalents of the participants (Henceforth $\mathrm{CE}$ ) from the experiment to reveal the shape of value and weighting function.

\subsection{Experiment 1}

\subsubsection{Experiment design}

Gonzalez and Wu (1999) study the shape of value and weighting function through the experiment. Based on their approach, we make some modifications to fit our IFPV framework and study the shape of value and weighting function under an intuitionistic fuzzy environment.

In order to assess individuals' value and the weighting function more accurately, we follow the traditional psychophysical paradigm. The basic design consists of 8 double-outcome gambling with 10 levels of the score function $s_{i}$ associated with the maximum outcomes. The two outcomes of gambling are (in dollars) $25-0,50-0,75-0,100-0,150-0,200-0,400-0$, 800-0. Note that all gambles offer nonnegative outcomes, so we just encode all of these outcomes as gains. To increase reliability, we fix the indeterminacy degree at 0.05 . Then the 10 $s_{i}$ levels are set as: $-0.93(0.01,0.94),-0.85(0.05,0.90),-0.75(0.10,0.85),-0.45(0.25,0.70)$, $-0.15(0.40,0.55), 0.05(0.5,0.45), 0.25(0.6,0.35), 0.55(0.75,0.20), 0.85(0.90,0.05), 0.93(0.94$, 0.01). (What's in the bracket are indeed the intuitionistic fuzzy numbers. Actually, the settings here can be extended to interval-valued intuitionistic fuzzy numbers instead. For the simplicity of illustration, we only show the intuitionistic fuzzy numbers in our experiment. The interval-valued vision of value function shape is just to estimate separately using two sides of interval-valued intuitionistic numbers. Therefore, we obtain a value function band instead).

In the questionnaire, the participants are asked to select a certainty equivalent to a gamble from the available options. For example, a gamble offers $50 \%$ chance to win $\$ 100,45 \%$ chance to win $\$ 0$ and $5 \%$ chance without any information. We then ask the participants which they 
prefer, sure amounts of 100, 80, 60, 40, 20 and 0 dollars or the gamble? For each row in Table 1, the participants check whether they prefer a sure amount to the gamble. For example, if a participant prefers a sure amount of $\$ 60$ to the gamble, but prefers the gamble to $\$ 40$ or less. The participant will place a check mark as follows:

Table 1. Choices table for gamble experiments

\begin{tabular}{|c|c|c|}
\hline Money (no gamble) & Prefer sure thing & Prefer gamble \\
\hline 100 & $\sqrt{ }$ & \\
\hline 80 & $\sqrt{ }$ \\
\hline 60 & $\sqrt{ }$ & \\
\hline 40 & & $\sqrt{ }$ \\
\hline 20 & & $\sqrt{ }$ \\
\hline 0 & & $\sqrt{ }$ \\
\hline
\end{tabular}

From the above response, we infer that the CE for this participant must be somewhere between $\$ 40$ and $\$ 60$. Thus, the participant needs to provide a precise threshold value when he/she will change the choice from prefer the sure amount to gamble. This value is the CE for each gamble. We can obtain all $80 \mathrm{CEs}$ as IFPV, to find the value function and the weighting function.

\subsubsection{Results and discussions}

The average $\mathrm{CE}$ for each of the 80 double-outcome gambles is shown in Table 2. They are average threshold values collected on 12 samples in each combination. And the estimations of the value function and weighting function are organized as follows:

Table 2. Average certainty equivalent for each gamble $(\mathrm{N}=12)$

\begin{tabular}{|c|c|c|c|c|c|c|c|c|c|c|}
\hline \multirow{2}{*}{ Outcomes } & \multicolumn{9}{|c|}{ Score function } \\
\cline { 2 - 11 } & $-\mathbf{0 . 9 3}$ & $-\mathbf{0 . 8 5}$ & $-\mathbf{0 . 7 5}$ & $-\mathbf{0 . 5 5}$ & $-\mathbf{0 . 1 5}$ & $\mathbf{0 . 0 5}$ & $\mathbf{0 . 2 5}$ & $\mathbf{0 . 5 5}$ & $\mathbf{0 . 8 5}$ & $\mathbf{0 . 9 3}$ \\
\hline $\mathbf{2 5 - 0}$ & $\mathbf{6 . 9 7 9 2}$ & 8.4792 & 9.2083 & 11.6042 & 13.0000 & 15.1250 & 17.0833 & 19.4792 & 20.7083 & 23.4583 \\
\hline $\mathbf{5 0 - 0}$ & 8.7500 & 10.2083 & 12.0833 & 15.8333 & 19.5833 & 24.1667 & 27.0833 & 33.1250 & 43.7500 & 44.7500 \\
\hline $\mathbf{7 5 - 0}$ & $\mathbf{1 3 . 7 5 0 0}$ & 14.0000 & 15.9167 & 24.0625 & 28.3333 & 33.1250 & 40.0000 & 46.7708 & 56.8750 & 62.5417 \\
\hline $\mathbf{1 0 0 - 0}$ & 13.3333 & 17.0833 & 17.9167 & 27.5000 & 35.7500 & 45.0000 & 52.9167 & 65.4167 & 78.1818 & 90.7500 \\
\hline $\mathbf{1 5 0}-\mathbf{0}$ & $\mathbf{2 2 . 1 6 6 7}$ & 23.3333 & 31.2500 & 38.9583 & 50.8333 & 62.0833 & 66.6667 & 86.4583 & 100.4167 & 115.9167 \\
\hline $\mathbf{2 0 0 - 0}$ & 26.8333 & 30.4167 & 36.2500 & 51.6667 & 79.1667 & 91.6667 & 99.1667 & 110.8333 & 133.3333 & 158.3333 \\
\hline $\mathbf{4 0 0 - 0}$ & 45.8333 & 49.5833 & 62.5000 & 95.0000 & 120.0000 & 155.8333 & 177.5000 & 206.6667 & 285.8333 & 295.0000 \\
\hline $\mathbf{8 0 0 - 0}$ & 99.1667 & 105.0000 & 137.5000 & 173.3333 & 248.3333 & 334.1667 & 363.3333 & 402.5000 & 531.6667 & 588.3333 \\
\hline
\end{tabular}

Firstly, we adopt nonparametric estimate method of Gonzalez and Wu (1999) without adding the fuzzy terms. We assume that the value function and the weighting function are: $v(X)=X^{\alpha}$ and $g\left(s_{i}\right)=\frac{\eta\left(s_{i}+1\right)^{\gamma}}{\eta\left(s_{i}+1\right)^{\gamma}+\left(1-s_{i}\right)^{\gamma}}$ respectively. We are going to estimate the param- 
eters $\eta, \gamma$ and $\alpha$ in equation: $y=\left(\frac{\eta\left(s_{i}+1\right)^{\gamma}}{\eta\left(s_{i}+1\right)^{\gamma}+\left(1-s_{i}\right)^{\gamma}}\right)^{\left(\frac{1}{\alpha}\right)}$. We show the estimated results in Table 3, and plot Figures 5 and 6 based on the results. From these two graphs, we can see that the shapes of functions are similar to those in Gonzalez and Wu (1999). And the corresponding values of parameters controlling the shapes are very close to those in Gonzalez and $\mathrm{Wu}$ (1999). This implies that the effects of weighting function are basically the same for our experiment. However, we find that the value of $\alpha$ is much higher than that in Gonzalez and $\mathrm{Wu}$ (1999). Because this is a fuzzy information-based experiment, if we treat it like only under uncertainty, not under ambiguity environment, the fuzzy contribution part will be miscalculated in the value function, which lead to a higher $\alpha$ than 0.49 .

Now, we add the fuzzy term in our estimation model based the intuitionistic fuzzy PT. After generating the random values of $\beta$, we are going to estimate the parameters $\eta, \gamma$ and $\alpha$ in equation: $y=\left(\frac{\eta\left(s_{i}+1\right)^{\gamma}}{\eta\left(s_{i}+1\right)^{\gamma}+\left(1-s_{i}\right)^{\gamma}}+\beta \pi_{i}\right)^{\left(\frac{1}{\alpha}\right)}$. After one thousand times of simulation, we obtain the following estimated parameter values: $\eta=0.7060, \gamma=0.4833$ and $\alpha=0.9021$. Compared with the parameters above, $\eta$ decreases, $\gamma$ increases, and $\alpha$ increases too (Figure 7 and Figure 8). From the value function, we find that after incorporating fuzzy information in decision making, the value of $\alpha$ remains below 1 , but it increases. This means that although people are still risk averse, they become less risk averse under an intuitionistic fuzzy environment than under uncertainty without ambiguity. A potential explanation is that when there is fuzzy information regarding the probabilities of different consequents of an event, people tend to be over-optimistic regarding the indeterminacy part. In other words, for the part of indeterminacy, they may arbitrarily assign higher possibilities to the positive prospects of the event, while at the same time, assign lower possibilities to the negative prospects. As a result, with the presence of fuzzy information, the expected payoff of an event, perceived by the decision-makers, is more likely to be overestimated. Hence, compared with the situation where there is no indeterminacy, risky choices may appear to be more attractive when there are certain degrees of indeterminacy. Because of the above over-optimistic arbitrary decision-making process, people become less risk averse when the degree of ambiguity rises under an intuitionistic fuzzy environment. Moreover, we can see, from Figure 8, that the weighting function curve with fuzzy information is almost parallel with the one without fuzzy information, but at a lower position close to the latter. This means that with the presence of ambiguity, people systemically underweight the probabilities of the outcomes with gains as well as those of the outcomes with losses.

Table 3. Estimated coefficients in Gonzalez and Wu's model without fuzzy terms

\begin{tabular}{|c|c|c|c|c|}
\hline \multicolumn{5}{|c|}{ Estimated coefficients } \\
\hline & Estimate & SE & t-Stat & p-Value \\
\hline$\eta$ & 0.80235 & 0.25117 & 3.1945 & 0.0020308 \\
\hline$\gamma$ & 0.46553 & 0.032781 & 14.201 & $3.306 \mathrm{e}-23$ \\
\hline$\alpha$ & 0.82274 & 0.17007 & 4.8377 & $6.6004 \mathrm{e}-06$ \\
\hline
\end{tabular}




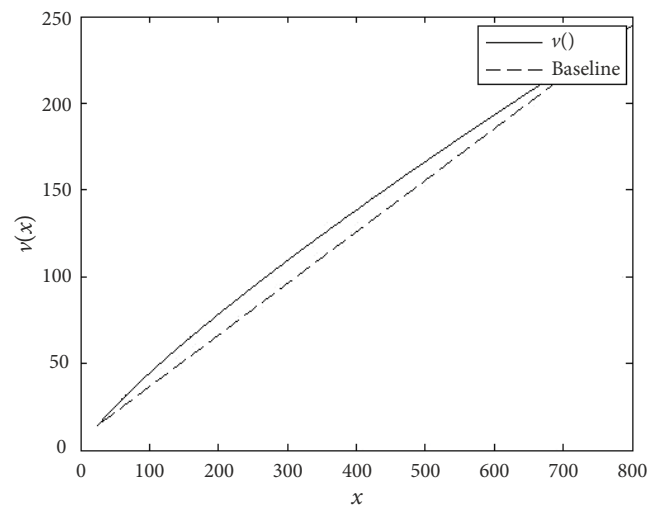

Figure 5. The value function in Gonzalez and Wu's model

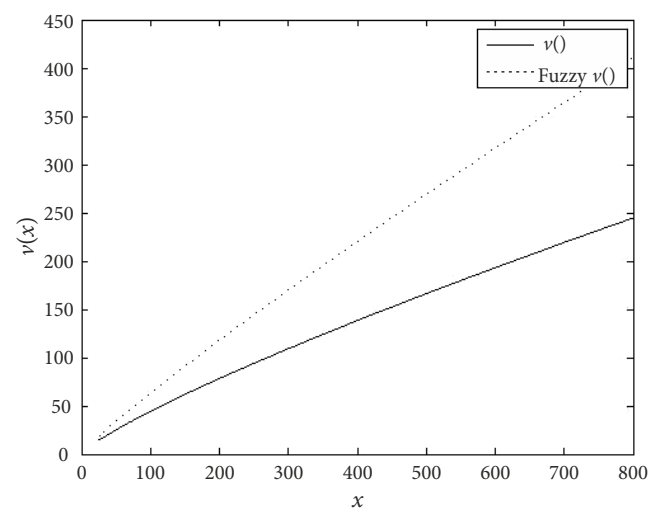

Figure 7. The value function under IFPT

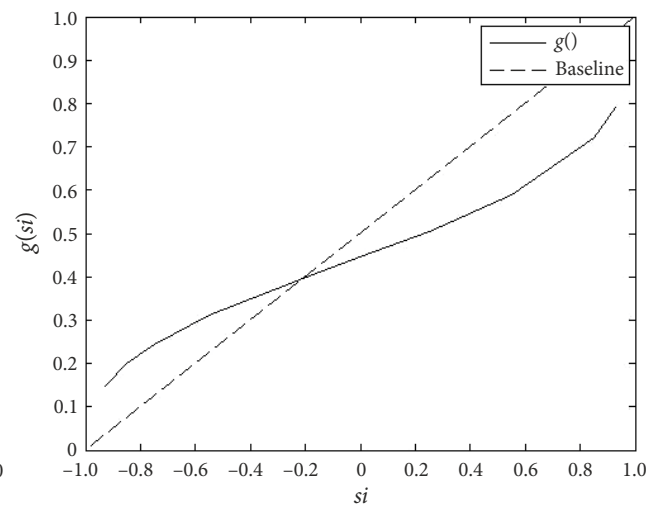

Figure 6. The weighting function in Gonzalez and Wu's model

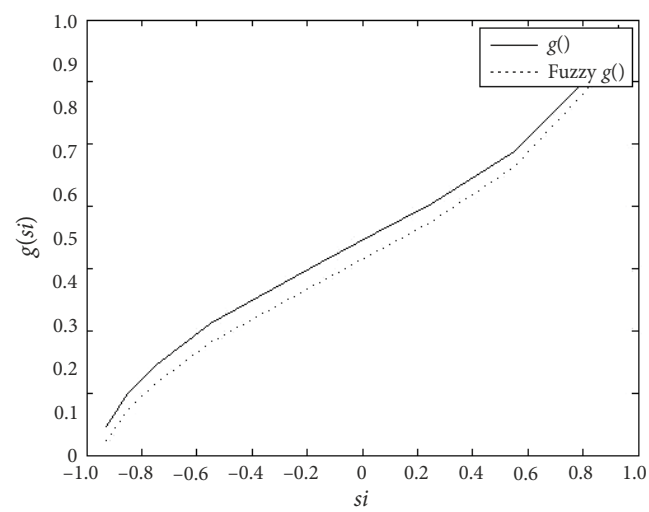

Figure 8. The weighting function under IFPT

\subsection{Experiment 2}

\subsubsection{Experiment design}

This experiment is designed to test the combination rules under an intuitionistic fuzzy environment. There are two sets of questions: the first set of questions is designed to test the addition principle; and those in the second set are designed to test the multiplication principle.

In group 1, participants are asked two questions about the index. There are two stocks related to this index. A rise in either of the stock prices will lead to an increase in the stock index. But the index falls if and only if the prices of both stocks decrease. The first entry in the cell indicates the probability that the stock price will rise (i.e., the membership of "rise"), and the second entry shows the probability of falling (i.e., the non-membership of "rise"). The degree of indeterminacy is given by: 1 minus the sum of the membership and the non-membership. For example, in Table 4, question 1, the price of stock 1 will rise with probability 0.6 and fall with probability 0.3 , so the degree of indeterminacy is given by: $1-(0.6+0.3)=0.1$. Here, the participants are asked to choose how they think the indexes would change, i.e., rise, fall or uncertain. This experiment is designed to examine how well people use the addition rule to derive another set of intuitionistic fuzzy information. 
Table 4. Questions in Group 1

\begin{tabular}{|c|c|c|c|}
\hline Group 1 & \multicolumn{2}{|c|}{ Fuzzy information for stocks } & Index \\
\hline Question 1 & $(0.6,0.3)$ & $(0.7,0.2)$ & $(0.88,0.06)$ (theoretical) \\
\hline Question 2 & $(0.3,0.5)$ & $(0.4,0.4)$ & $(0.58,0.20)$ (theoretical) \\
\hline
\end{tabular}

Table 5. Questions in Group 2

\begin{tabular}{|c|c|c|c|}
\hline Group 2 & \multicolumn{2}{|c|}{ Fuzzy information for two stages } & Success rate of the project \\
\hline Question 1 & $(0.5,0.3)$ & $(0.6,0.2)$ & $(0.30,0.44)$ (theoretical) \\
\hline Question 2 & $(0.4,0.5)$ & $(0.3,0.6)$ & $(0.12,0.80)$ (theoretical) \\
\hline
\end{tabular}

In group 2, Table 5 shows two questions about an investment project involving two stages. This project will be successful accomplished only if both stages are completed successfully.

The fuzzy information in the table above is the success rate for each stage. The first entry in each cell shows the probability of success (i.e., the membership of "success"), and the second one is the probability of failure (i.e., the non-membership of "success"). The degree of indeterminacy is given by: 1 minus the sum of the membership and the non-membership. For example, in Table 5, question 1, the first stage of the project will be a success with the probability 0.5 , or a failure with the probability 0.3 , and the degree of indeterminacy is given by: $1-(0.5+0.3)=0.2$. Here, the participants are asked to decide whether the project will be successful, based on two stages of fuzzy information. The purpose of this experiment is to examine how well people use the multiplication rule to derive the set of intuitionistic fuzzy information.

\subsubsection{Results and discussions}

Based on the choices of 12 participants in the experiment, we get the experimental data to illustrate their application the addition principle and the multiplication principle under a fuzzy environment. As shown in Table 6, although there is a slight difference between the experimental results and our theoretical predictions, the participants' choices under an intuitionistic fuzzy environment are consistent with the addition and multiplication principles of our framework.

Table 6. Results in the addition principle and the multiplication principle

\begin{tabular}{|l|c|c|c|c|}
\hline $\begin{array}{l}\text { Fuzzy } \\
\text { formulae }\end{array}$ & $(0.6,0.3)+(0.7,0.2)$ & $(0.3,0.5)+(0.4,0.4)$ & $(0.5,0.3) *(0.6,0.2)$ & $(0.4,0.5) *(0.3,0.6)$ \\
\hline Theoretical & $(0.88,0.06)$ & $(0.58,0.2)$ & $(0.3,0.44)$ & $(0.12,0.8)$ \\
\hline $\begin{array}{l}\text { Experiment } \\
\text { results }\end{array}$ & $(0.92,0)$ & $(0.42,0.33)$ & $(0.25,0.42)$ & $(0.08,0.83)$ \\
\hline
\end{tabular}




\section{Conclusions and discussions}

This paper develops a generalized decision-making framework based on the prospect theory under an intuitionistic fuzzy environment, by closely integrating the prospect theory and the intuitionistic fuzzy sets into our framework. We demonstrate how to compute the intuitionistic fuzzy prospect value as the reference values for decision-making and elaborate a four-step editing phase and a valuation phase with two key functions: the value function and the weighting function.

We conduct experiments to test the validity and properties of our decision-making framework. The experiments reveal that the ways that subjects choose to deal with information are consistent with what are implied by the fuzzy logic of our framework in various scenarios. The experimental results show that (i) the shapes of the value function and the weighting function in our framework are in line with those of prospect theory, (ii) people are less risk averse when making decisions under an intuitionistic fuzzy environment than under uncertainty, and (iii) with the presence of ambiguity, people systemically underweight the probabilities of the outcomes with gains as well as those of the outcomes with losses.

Our methodology can elicit prospects not only under uncertainty but also under ambiguity, and the decision-making patterns can be fully captured by parameters in the value function and the weighting function. Our framework offers an illuminating approach for decision-making under an intuitionistic fuzzy environment. It can be applied in a wide range of decision-making problems, including those of the multi-attribute decision-making. It is not only an extension of PT, but also a further application of the theory of intuitionistic fuzzy sets in a more general environment. As our approach generalizes to the case of ambiguity, the prospect theory can therefore be viewed as a special case in our framework.

\section{Acknowledgements}

This research was funded by the National Natural Science Foundation of China (No. 71401116, No. 71571123, No. 71473168), and by Sichuan University (No. 2018hhs-07, No. YJ201353, No. Skyb201404, No. skqy201766).

\section{References}

Allais, M. (1953). Le comportement de i'homme rationnel devant le risque: critique des postulats et axiomes de i'cole americaine. Econometrica, 21(4), 503-546. https://doi.org/10.2307/1907921

Andrade, R. A. E., González, E., Fernández, E., \& Gutiérrez, S. M. (2014). A fuzzy approach to prospect theory. In R. Espin, R. Pérez, A. Cobo, J. Marx, \& A. Valdés. (Eds.), Soft computing for business intelligence. Studies in computational intelligence (Vol. 537). Berlin, Heidelberg: Springer. https://doi.org/10.1007/978-3-642-53737-0_3

Atanassov, K. T. (1986). Intuitionistic fuzzy sets. Fuzzy Sets and Systems, 20(1), 87-96. https://doi.org/10.1016/S0165-0114(86)80034-3

Atanassov, K. T., \& Gargov, G. (1989). Interval valued intuitionistic fuzzy sets. Fuzzy Sets and Systems, 31(3), 343-349. https://doi.org/10.1016/0165-0114(89)90205-4

Chen, S. M., \& Tan, J. M. (1994). Handling multicriteria fuzzy decision-making problems based on vague set theory. Fuzzy Sets \& Systems, 67(2), 163-172. https://doi.org/10.1016/0165-0114(94)90084-1 
Edwards, K. D. (1996). Prospect theory: a literature review. International Review of Financial Analysis, 5(1), 19-38. https://doi.org/10.1016/S1057-5219(96)90004-6

Gao, J., \& Liu, H. (2016). A new prospect projection multi-criteria decision-making method for interval-valued intuitionistic fuzzy numbers. Information, 7(4), 64.

Gonzalez, R., \& Wu, G. (1999). On the shape of the probability weighting function. Cognitive Psychology, 38(1), 129-166. https://doi.org/10.1006/cogp.1998.0710

Kahneman, D., \& Tversky, A. (1979). Prospect theory: an analysis of decision under risk. Econometrica, 47(2), 140-170. https://doi.org/10.2307/1914185

Krohling, R. A., \& Souza, T. T. M. D. (2012). Combining prospect theory and fuzzy numbers to multicriteria decision-making. Expert Systems with Applications, 39(13), 11487-11493. https://doi.org/10.1016/j.eswa.2012.04.006

Leclerc, F., Schmitt, B. H., \& Dubé, L. (1995). Waiting time and decision-making: is time like money? Journal of Consumer Research, 22(1), 110-119. https://doi.org/10.1086/209439

Levy, M., \& Levy, H. (2002). Prospect theory: much ado about nothing? Management Science, 48(10), 1334-1349. https://doi.org/10.1287/mnsc.48.10.1334.276

Li, P., Liu, S. F., \& Zhu, J. J. (2012). Intuitionistic fuzzy stochastic multi-criteria decision-making methods based on prospect theory. Control and Decision, 27(11), 1601-1606.

Li, P., Yang, Y., \& Wei, C. (2017). An intuitionistic fuzzy stochastic decision-making method based on case-based reasoning and prospect theory. Mathematical Problems in Engineering, 6, 1-13. https://doi.org/10.1155/2017/2874954

Li, X. H. (2013). Intuitionistic trapezoidal fuzzy multi-attribute decision-making method based on cumulative prospect theory and Choquet integral. Application Research of Computers, 30(8), 24222425.

Li, X. H., \& Chen, X. H. (2014). Extension of the TOPSIS method based on prospect theory and trapezoidal intuitionistic fuzzy numbers for group decision-making. Journal of Systems Engineering and Electronics, 23(2), 231-247. https://doi.org/10.1007/s11518-014-5244-y

Liu, H. W., \& Wang, G. J. (2007). Multi-criteria decision-making methods based on intuitionistic fuzzy sets. European Journal of Operational Research, 179(1), 220-233. https://doi.org/10.1016/j.ejor.2006.04.009

Liu, P., Jin, F., Zhang, X., Su, Y., \& Wang, M. (2011). Research on the multi-attribute decision-making under risk with interval probability based on prospect theory and the uncertain linguistic variables. Knowledge-Based Systems, 24(4), 554-561. https://doi.org/10.1016/j.knosys.2011.01.010

Mayer, P. C. (1995). Electricity conservation: consumer rationality versus prospect theory. Contemporary Economic Policy, 13(2), 109-118. https://doi.org/10.1111/j.1465-7287.1995.tb00747.x

Meng, F., Tan, C., \& Chen, X. (2015). An approach to Atanassov' interval-valued intuitionistic fuzzy multi-attribute decision making based on prospect theory. International Journal of Computational Intelligence Systems, 8(3), 591-605. https://doi.org/10.1080/18756891.2015.1036224

Newman, D. P. (1980). Prospect theory: implications for information evaluation. Accounting Organizations and Society, 5(2), 217-230. https://doi.org/10.1016/0361-3682(80)90011-2

Payne, J. W., Laughhunn, D. J., \& Crum, R. (1984). Multiattribute risky choice behavior: the editing of complex prospects. Management Science, 30(11), 1350-1361. https://doi.org/10.1287/mnsc.30.11.1350

Rieger, M. O., Wang, M., \& Hens, T. (2015). Risk preferences around the world. Management Science, 61(3), 637-648.

Sebora, T. C., \& Cornwall, J. R. (1995). Expected utility theory vs prospect theory: implications for strategic decision makers. Journal of Managerial Issues, 7(1), 41-61.

Tversky, A., \& Fox, C. R. (1995). Weighing risk and uncertainty. Psychological Review, 102(2), 269-283. https://doi.org/10.1037/0033-295X.102.2.269 
Tversky, A., \& Kahneman, D. (1986). Rational choice and the framing of decisions. Journal of Business, 59(4), 251-278.

Tversky, A., \& Kahneman, D. (1992). Advances in prospect theory: cumulative representation of uncertainty. Journal of Risk and Uncertainty, 5(4), 297-323. https://doi.org/10.1086/296365

Von Neumann, J. L., \& Morgenstern, O. V. (1953). The theory of games and economic behavior. Princeton: University Press.

Wang, J. Q., \& Zhang, Z. (2009). Aggregation operators on intuitionistic trapezoidal fuzzy number and its application to multi-criteria decision-making problems. Journal of Systems Engineering and Electronics, 20(2), 321-326.

Wang, J. Q., \& Nie, R. R. (2012). Multi-criteria group decision-making method based on intuitionistic trapezoidal fuzzy information. System Engineering Theory and Practice, 32(8), 1747-1753.

Wu, G., \& Gonzalez, R. (1999). Nonlinear decision weights in choice under uncertainty. Management Science, 45(1), 74-85. https://doi.org/10.1287/mnsc.45.1.74

$\mathrm{Xu}, \mathrm{Z}$. S., \& Xia, M. M. (2011). Induced generalized intuitionistic fuzzy operators. Knowledge-Based Systems, 24(2), 197-209. https://doi.org/10.1016/j.knosys.2010.04.010

$\mathrm{Xu}, \mathrm{Z}$. S. (2007). Intuitionistic fuzzy aggregation operators. Information Fusion, 14(1), 108-116.

Xu, Z. S., \& Cai, X. Q. (2012). Intuitionistic fuzzy information aggregation: theory and applications. Berlin: Springer-Verlag.

Zadeh, L. A. (1965). Fuzzy sets. Information and Control, 8(3), 338-353. https://doi.org/10.1016/S0019-9958(65)90241-X 